\title{
Evaluation of $F . x$ ananassa intra-specific and inter-specific back-crosses to generate new genetic material with increased fruit nutritional quality
}

\author{
Jacopo Diamanti $^{\mathrm{a}}$, Franco Capocasa ${ }^{\mathrm{a}}$, Maurizio Battino ${ }^{\mathrm{b}}$ and Bruno Mezzetti ${ }^{\mathrm{a}, *}$ \\ ${ }^{a} S A P R O V$ - Department of Environmental and Crop Science, Faculty of Agriculture, Università \\ Politecnica delle Marche, Ancona, Italy \\ ${ }^{\mathrm{b}}$ Department of Biochemistry, Biology \& Genetics, Medical School, Università Politecnica delle \\ Marche, Ancona, Italy
}

\begin{abstract}
During the last few years, the nutritional value of fruit has been widely studied and requested by consumers, especially for the general health benefits it can provide. Some of these benefits can be ascribed to the antioxidant capacity of fruit. Both quality and antioxidant attributes are currently considered as good tools to describe the nutritional quality of fruit.

In this study, offspring from inter and intra-specific strawberry cross combinations were studied with the aim of evaluating and identifying new genotypes that can show the best compromise between quality and nutritional characteristics. Parents and offspring populations were studied by analyzing fruit nutritional quality taking in account the following parameters: Total antioxidant capacity, total phenol and anthocyanin content. Eighty strawberry offspring, originating from 8 Fragaria spp. inter and intraspecific crosses, were screened to study the role of the genetic origin of the parents in segregating new genotypes with an improved nutritional quality of the fruit.

Strawberry progenies having $F$. virginiana glauca as a common parent showed a significant enhancement of their fruit quality and nutritional features confirming the interest that wild species are useful in improving several characteristics in cultivated strawberries.
\end{abstract}

Keywords: Strawberry, F. virginiana spp. glauca, antioxidant capacity, phenols, anthocyanins, breeding methods

\section{Introduction}

A greater consumption of fruit and vegetables is considered as one way of increasing the intake of antioxidants, and the strawberry, like other berries, represents one of the most important sources of bioactive compounds with antioxidant activity $[2,12,15,16,19,21]$. Accordingly, the increase of consumption of berries richer in "healthy compounds" is seen as an appropriate strategy for improving human health.

Increase of the antioxidant levels in fruit through breeding and/or biotech is an important option to support a higher antioxidant intake even when the consumption of fruit is low. If nutritional components are also combined with a high standard sensorial fruit quality the perspective of consumer health can be further improved by increased consumption [16]: This can be the case for strawberry fruit [23].

*Corresponding author: Bruno Mezzetti, Department of Environmental and Crop Science, Faculty of Agriculture, Università Politecnica delle Marche, Via Brecce Bianche 60100, Ancona, Italy. E-mail: b.mezzetti@univm.it. 
The breeding approach can succeed if the variability and heritability of the Total Antioxidant Capacity (TAC) trait indicate the possibility of achieving a breeding progress. The availability of genetic diversity within compatible species of any given crop will enhance the extent of improvement [3]. The biotechnological approach is now an integrative option to extend this improvement, but it is related to the knowledge of molecular tools able to promote more general increases in several metabolites through the modification of specific biosynthetic pathways [9]. However, the success of both breeding and biotech approaches is related to deep knowledge of the most useful wild and cultivated genetic diversity to be used in genetic and genomic studies.

The effect of genotypes in affecting the nutritional quality of strawberries is well known [1, 14, 18, 25], the levels of antioxidants and antioxidant capacity in strawberry extracts from whole fruits vary considerably among genotypes $[18,19,24]$ but few genotypes are well characterized for these important features. Furthermore, limited knowledge is available on the possibility of improving strawberry nutritional traits by breeding $[4,5]$. Some results have been found for berries and a moderate heritability for TAC, Total Phenols Content (TPH) and Anthocyanins Content (ACY) was demonstrated in blueberries and raspberries [6-8, 26, 27].

Accessions of the progenitor wild species are valued by strawberry breeders as sources of novel traits, especially pest resistance and abiotic stress tolerance [11]. Furthermore, previous investigations by the authors $[4,5,18,21]$ have shown improvement in fruit nutritional quality in breeding material originated by using Fragaria virginiana spp. glauca (FVG) as parent. This result is also supported by the study carried out by Wang and Lewers [24] showing that fruit of $F$. virginiana accessions had significantly higher antioxidant capacity, total phenolics, and total anthocyanins than fruit from F. chiloensis and F. x ananassa accessions.

A breeding program was developed to compare and evaluate different types of $F$. $x$ ananassa intra-specific and $F . x$ ananassa per FVG inter-specific back-crosses to generate new progenies to be selected for increased fruit nutritional. The program was designed on the basis of the above mentioned studies as well as following the indication that as few as three backcrosses can yield selections of cultivar quality [11].

With this aim the nutritional quality of strawberries was studied by analyzing fruit TAC. TPH and ACY of 80 strawberry offspring derived from 8 families: Two originated from $F$. $x$ ananassa intra specific crossing, three from back crossing of $\mathrm{F} 1-\mathrm{FVG} \times F$. $x$ ananassa and three originated from back crossing of $\mathrm{F} 2-\mathrm{FVG} \times F$. $x$ ananassa.

\section{Materials and methods}

\subsection{Chemicals}

Methanol 99\% ACS-ISO for analysis was purchased from Carlo Erba Reagenti (Milano, IT). Folin-Ciocalteu's reagent, sodium carbonate anhydrous, potassium chloride, sodium acetate, chloridric acid, glacial acetic acid, dihydrogen potassium phosphate, dipotassium hydrogen phosphate, 2,2'-azino-bis(3-ethylbenzothiazoline-6-sulphonic acid) diammonium salt $\left(\mathrm{ABTS}^{\bullet+}\right)$, 6-hydroxy-2,5,7,8-tetramethylchromane-2-carboxylic acid (Trolox), potassium persulfate, 3,4,5-trihydroxybenzoic acid (gallic acid) were purchased from Sigma-Aldrich (Sigma-Aldrich s.r.l., Milano, Italia).

\subsection{Plant genetic material}

Genetic variability, originated by 8 different strawberry intra and inter-specific cross combinations, was explored by analyzing 80 seedlings obtained from the following cross-combinations: 2 from intra-specific cross combinations between commercial cultivar and advanced selection of $F$. $x$ ananassa; $3 \mathrm{~F} 2$ back-crossing using the $\mathrm{F} 1$ selection AN94,414,52, $F$. $x$ ananassa (Don) $\times F$. virginiana glauca $(\mathrm{FVG} 22)$; and $3 \mathrm{~F} 3$ back-crossing using AN00,239,55 as common progenitor, an F2 selection derived from the back crossing of selection F1 AN94,414,52 with 91,143,5 ( $F$. $x$ ananassa advanced selection) (Table 1). The pedigree of the parents used in the cross combinations are reported in Table 2.

During one season (2009), the 80 seedlings (single plant) from the 8 families (from 8 to 11 seedlings for each family) were grown in open field conditions, at the P. Rosati University experimental farm, Agugliano (Ancona, IT), using the plastic hill culture production system. Fruits were hand harvested at a ripe stage (full red fruit from the third 
Table 1

List of families and corresponding progenitor and number of offspring taken into account for the study of fruit nutritional quality

\begin{tabular}{|c|c|c|c|c|c|}
\hline \multirow[t]{2}{*}{$\overline{\text { Families }}$} & \multicolumn{3}{|c|}{ Progenitors } & \multirow[t]{2}{*}{ No. of offspring } & \multirow[t]{2}{*}{ Type of crossing } \\
\hline & Mother & & Father & & \\
\hline AN07,003 & AN94,414,52 & $\mathrm{X}$ & AN99,78,51 & 11 & F2 Backcross \\
\hline AN07,004 & AN94,414,52 & $\mathrm{X}$ & ALBA & 10 & F2 Backcross \\
\hline AN07,005 & AN94,414,52 & $\mathrm{X}$ & CLERY & 9 & F2 Backcross \\
\hline AN07,006 & AN00,239,55 & $\mathrm{X}$ & AN99,78,51 & 10 & F3 Backcross \\
\hline AN07,007 & AN03,338,56 & $\mathrm{X}$ & AN99,78,51 & 10 & Intra-specific \\
\hline AN07,009 & CLERY & $\mathrm{X}$ & AN99,78,51 & 11 & Intra-specific \\
\hline AN07,215 & AN00,239,55 & $\mathrm{X}$ & AN03,338,56 & 8 & F3 Backcross \\
\hline AN07,216 & AN00,239,55 & $\mathrm{X}$ & CLERY & 11 & F3 Backcross \\
\hline
\end{tabular}

Table 2

List of the pedigree of parents involved in cross combinations

\begin{tabular}{llll}
\hline Genotype & \multicolumn{3}{l}{ Progenitors } \\
\cline { 2 - 4 } & Mother & Father \\
\hline ALBA & TUDLA & MISS \\
CLERY & SWEET CHARLIE & $X$ & MARMOLADA ONEBOR \\
AN99,78,51 & $95,617,1$ & $X$ & DARSELECT \\
AN03,338,56 & SVEVA & $X$ & PATTY \\
AN94,414,52 & DON & $X$ & FVG 22 \\
AN00,239,55 & AN94,414,52 & $X$ & $91,143,5$ \\
\hline
\end{tabular}

and fourth harvest); sorted to eliminate damaged, shriveled, and unripe fruit; and selected for uniform size and color. The fruit harvested from each plant of any genotype were kept separate and stored at $-20^{\circ} \mathrm{C}$ until used for assays.

Data from each family were compared with those of the corresponding parents.

\subsection{Strawberry fruit extraction method}

From a pool of fruits from both harvests, stored at $-20^{\circ} \mathrm{C}$, ten homogenously colored fruits were sampled and two specular slices were cut from each fruit which were then minced into small pieces, $10 \mathrm{~g}$ of this mixture was weighed and placed in a tube to be extracted. The pieces of strawberry were extracted with methanol (1:4, fruit: Methanol, $\mathrm{w} / \mathrm{w})$ and the extraction procedure was divided into two parts.

The first extraction consisted of $20 \mathrm{ml}$ methanol used to homogenize the fruit pieces in an Ultraturrax T25 homogenizer (Janke and Kunkel, IKA Labortechnik, Staufen, DE). The homogenized suspension was placed in continuous agitation (or ultrasound assisted) for $30 \mathrm{~min}$ and the extraction took place in the dark. The suspension was centrifuged at $4500 \mathrm{~g}$ for $10 \mathrm{~min}$ (Centrifuge Rotofix32, Hettich Zentrifugen, Tuttlingen, DE) and the recovered supernatant was collected and stored in three separate amber vials which will correspond to three replications for subsequent analysis.

The second part of the procedure was carried out adding $20 \mathrm{ml}$ more of methanol to the already grinded, extracted and pelleted fruit for a more complete and extensive extraction. The pellet was re-suspended and the procedure repeated as above. The supernatant was collected, joining the previous ones in the same amber vials and stored at $-20^{\circ} \mathrm{C}$.

\subsection{TAC}

TAC was evaluated by the ABTS assay, according to a previously validated procedure [13, 17]. ABTS, a chromogen and colorless substance, is changed into its colored monocationic radical form (ABTS ${ }^{\bullet+}$ ) by an oxidative agent. 
Addition of antioxidants reduces $\mathrm{ABTS}^{\bullet+}$ into its colorless form. The extent of decolorization as percentage of inhibition of $\mathrm{ABTS}^{\bullet+}$ is determined as a function of concentration and calculated relative to the reactivity of Trolox, a water-soluble vitamin E analogue. Antioxidant activity was expressed as mg of Trolox equivalent per $\mathrm{kg}$ of fresh pulp weight. The calibration was calculated as linear regression from the dose response Trolox Standard. The results are expressed as mg of Trolox equivalent per $\mathrm{kg}$ of Fresh fruit.

\section{5. $T P H$}

TPH was evaluated by the Folin-Ciocaltou's reagent method [20] using gallic acid as standard for the calibration curve. Results were calculated and expressed as $\mathrm{mg}$ of gallic acid per kilogram of fresh fruit. Value of standards ranged from 10 to $50 \mathrm{mg}$ gallic acid/L. Briefly, A test tube (glass) was filled with $7.0 \mathrm{ml}$ water. Afterwards, $1 \mathrm{~mL}$ of the diluted sample $(1: 20)$ was added, followed by the addition of $500 \mu \mathrm{L}$ Folin-Ciocalteu-Reagent and vortexed. After $3 \mathrm{~min} 1.5 \mathrm{~mL}$ sodium carbonate $(0.53 \mathrm{~mol} / \mathrm{l})$ was added and the tube was mixed once more and then stored in the dark for $60 \mathrm{~min}$. The absorbance of the sample was measured after exactly $60 \mathrm{~min}$ at $760 \mathrm{~nm}$.

\subsection{ACY assay}

ACY assay was measured by the pH differential shift method [10]. The assay was performed using the anthocyanins' characteristic to change intensity of hue depending on $\mathrm{pH}$ shifting. Briefly, the samples were diluted to a ratio of $1: 10$ with potassium-chloride $(\mathrm{pH} 1.00)$ and with sodium acetate $(\mathrm{pH} 4.50)$ and the corresponding maximum absorbance for both solutions was measured (respectively at $\lambda=500 \mathrm{~nm}$, approximately and $\lambda=700 \mathrm{~nm}$ ). To calculate the content of anthocyan in the following formula was used:

$$
\text { mg Pel-3-glu/kg FW }=\frac{\left[\left(A_{\lambda \max }-A_{700}\right)_{\mathrm{pH} 1.00}-\left(A_{\lambda \max }-A_{700}\right)_{\mathrm{pH} 4.50}\right] \times M W \times F \times 1000}{\varepsilon \times d \times E}
$$

$A=$ absorbance $M W=$ molecular weight of pelargonidin-3-glucoside $=433.2[\mathrm{~g} / \mathrm{mol}] ; F=$ dilution factor $=10 ; d=$ cell pathlengths $[\mathrm{cm}] ; \varepsilon=$ molar absorbance of Pel-3-glu $=15600\left[\mathrm{~L} /\left(\mathrm{mol}^{*} \mathrm{~cm}\right)\right] ; E=$ sample weight $[\mathrm{kg} / \mathrm{L}$ extracting agent $] ; 1000=$ Factor for $\mathrm{mg}$.

The results were expressed as mg of pelargonidin-3-glucoside (the compounds more representative for anthocyanins in strawberry) per kilogram of fresh weight (mg of Pel-3-Glu/Kg FW).

\subsection{Experimental trial and statistical analysis}

Nutritional quality analyses were performed during the last harvesting season (2009), in triplicate for each genotype of fruit sample. All the data were analysed using one-way analysis of variance, ANOVA test for mean comparisons. The ANOVA of families was performed by analysing the effect of type of intra-inter-specific crossing, crossing combinations and offspring. Data on fruit nutritional analyses are referred to the mean of the three harvest times and are reported as mean \pm standard deviation (SD). The differences were calculated according to the Student Newman Keuls (SNK) test, and were considered significant at $p \leq 0.05$. Correlations were calculated on a genotype mean basis. Normal Distribution were calculated on the observation resulting from nutritional quality analyses. All analyses were performed using STATISTICA (Statsoft Inc., Tulsa).

\section{Results}

\subsection{Offspring total distribution and family variation of fruit TAC}

Distribution analysis was carried out on all the 80 seedlings descending from 8 cross combinations from two intraspecific crossing of $F$. $x$ ananassa, three F1 of FVG backcrossing and three F2 of FVG back crossing. TAC values of all offspring populations resulted in a normal distribution (Fig. 1), with the highest frequency (36 accessions) corresponding to the value of $23.28 \mu \mathrm{mol} \mathrm{TE} / \mathrm{g}$ FW (Fig. 1), this matching with the TAC values of 3 parents (Clery; 


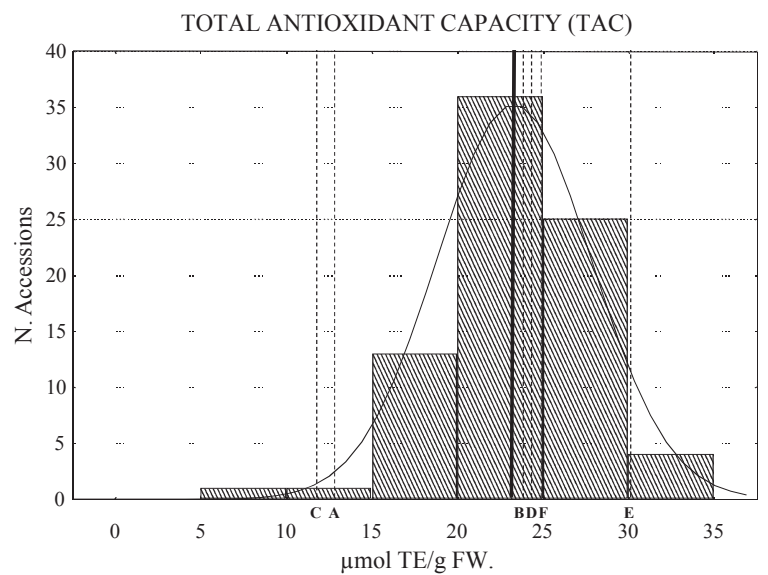

Fig. 1. Distribution of fruit Total Antioxidant Capacity of all offspring originated from the eight cross combinations and Normal Distribution calculated with Kolmogorov-Smirnov test $d=0.047, p=$ n.s. $(p \leq 0.05)$. Letters correspond to the mean value of each parents: A AN99,78,51; B Clery; C Alba; D AN94,414,52; E AN00,239,55; F AN03,338,56.

AN94,414.52; AN03,338,56). Only 4 accessions had TAC values higher than AN00,239,55, the only parent with the highest TAC value, while only two accessions had the lowest TAC values, even lower than the parents Alba and AN99,78,51.

TAC values among families resulted different (ANOVA $p \leq 0.01$ ). The highest TAC mean value, expressed as $\mu \mathrm{mol} \mathrm{TE} / \mathrm{g} \mathrm{FW}$, was found for family AN07,216 (28.12 \pm 0.48$)$ which was significantly higher than those of other families. High mean values were also detected in family AN07,215 (26.70 \pm 0.31$)$. These F3 families, originated from the F2 - FVG combined with Clery (AN07,216) and with AN03,338,56 (AN07,215), performed with a large variation of TAC values; offsprings mean variation of family AN07,216 remained between the parent's values but seedlings few resulted above the parent known for the highest TAC value, the F2 selection AN00,239,55 (Fig. 1).

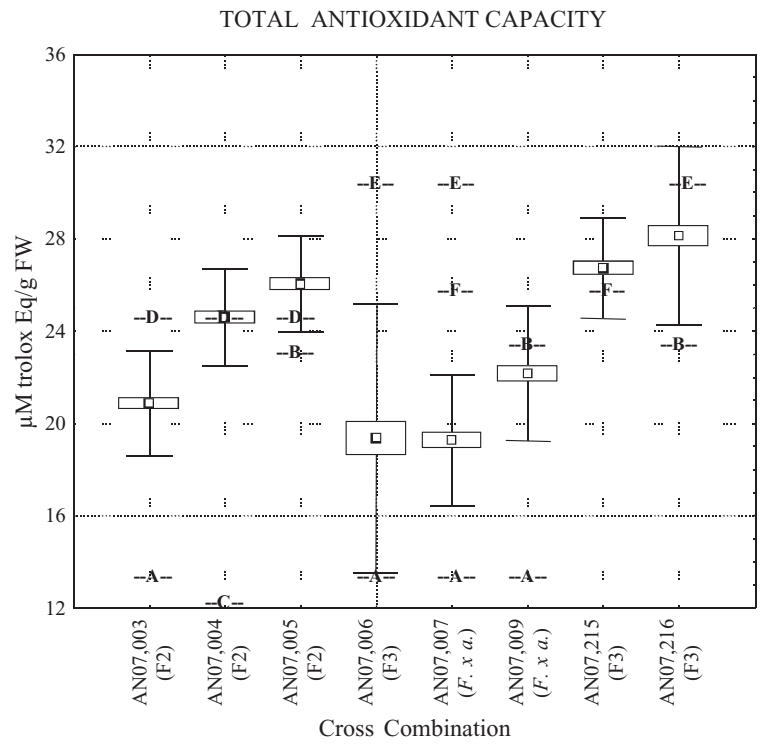

Fig. 2. Distribution expressed as Mean, Standard Error and Standard Deviation, calculated by Box and Whiskers method (Statistica, Statsoft) for each cross combination for fruit Total Antioxidant Capacity. Letters correspond to the mean value of each parents: A AN99,78,51; B Clery; C Alba; D AN94,414,52; E AN00,239,55; F AN03,338,56. 
On the contrary, AN07,006, the other F3 family with AN99,78,51 as $F$. x ananassa parent performed with the lowest TAC values $(19.34 \pm 0.75)$.

F2 offsprings from families AN07,005 (26.03 \pm 0.28$)$ and AN07,004 (24.58 \pm 0.27$)$ also performed with high TAC values of their fruit. Offspring mean TAC value of AN07,005 was higher than the values of the corresponding parents; while mean values of the other families corresponded to the parent means. These results highlight that using F. $x$ ananassa parents selected for high TAC values of their fruit can give origin to a great variability of this important trait with improvement compared to the parents. In particular, Alba and Clery showed a positive combination with F1 - FVG parent, while AN07,003, the third F2 family, differed for lower mean values ( $20.85 \pm 0.28$ ). In this case, the selection AN99,78,51 resulted in a negative genetic effect for this important trait also when used in intra-specific cross combinations. In fact, fruit from intra-specific crosses having this selection as parent performed with lower TAC mean values, as for AN07,009 (22.16 \pm 0.36$)$ and in particular for AN07,007 (19.27 \pm 0.36$)$. Moreover, when a comparison was made with the four cross combinations having AN99,78,51 as male parent, the negative effect of this genetic background clearly appeared on decreasing fruit antioxidant capacity.

\subsection{Offspring total distribution and family variation of fruit TPH}

Statistical analysis on offsprings total distribution for TPH resulted as non significant. The highest frequency fell below all parents (Fig. 3). However, fruit of 14 offspring was found to have TPH values higher than 5 parents, but none went beyond the highest value detected for AN00,239,55.

The ANOVA analyses for TPH showed significant differences among cross combinations as for TAC.

F3 families emerged with the highest values for TPH, thus confirming the results observed for the TAC analyses. In fact, the highest TPH means were detected for families AN07,216 (3.07 \pm 0.08$)$ and AN07,215 (3.03 \pm 0.05$)$, both of them originated from the back crossing of $\mathrm{F} 2-\mathrm{FVG}$ with F. $x$ ananassa (Fig. 4). Those two cross combinations have shown dissimilar variability; in fact, family AN07,216 differed for a higher variability among offspring, some even below AN03,338,56, the parent of fruit with lowest TPH and none able to go beyond AN00,239,55 the parent of fruit with highest TPH. Offspring of family AN07,215 showed a lower variation comprised in the parent TPH values. As already observed for fruit TAC, offspring of family AN07,006 have shown the lowest mean values for TPH $(1.71 \pm 0.04)$ among all cross combinations and with a variation even below the two parents. These data confirmed the unhelpful effect of AN99,78,51 as parent to be used to improve strawberry fruit nutritional quality.

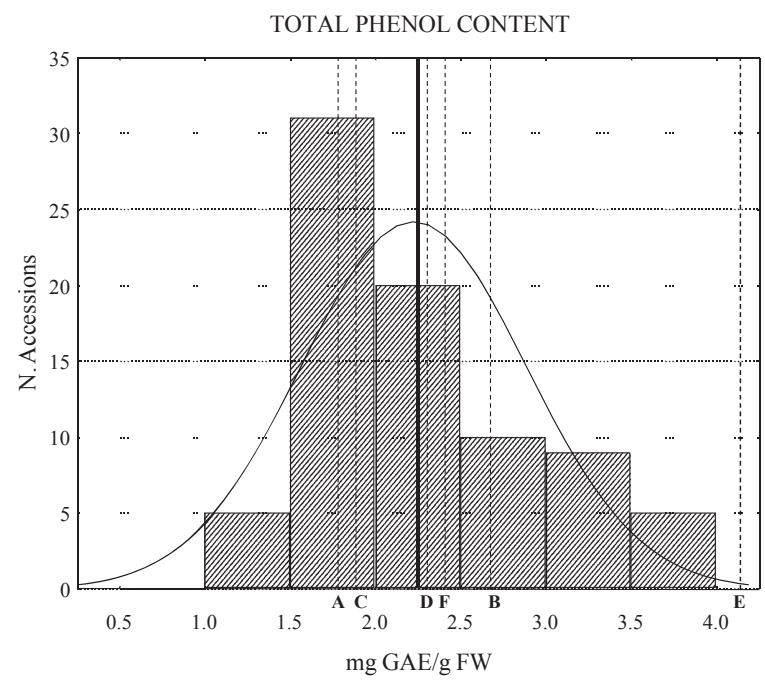

Fig. 3. Distribution detected for the fruit Total Phenol Content of offspring originated from the eight cross combination and the Normal Distribution calculated with Kolmogorov-Smirnov test $d=0.113, p=$ n.s. $(p \leq 0.05)$. Letters correspond to the mean value of each parents: A AN99,78,51; B Clery; C Alba; D AN94,414,52; E AN00,239,55; F AN03,338,56. 


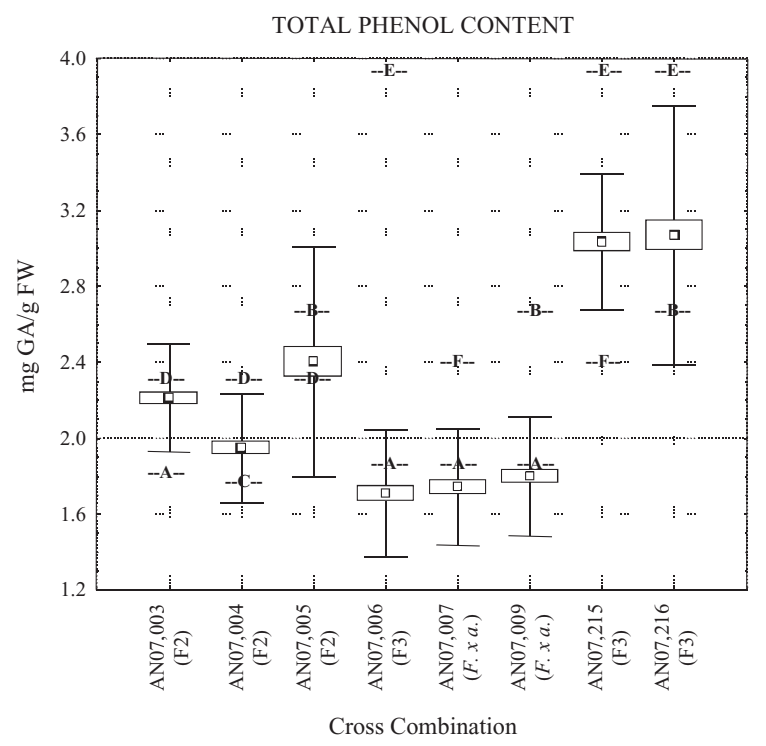

Fig. 4. Box and whisker Cross Combination Total Phenol Content. Results are shown as mean value, Standard Error, Standard Deviation. Letters correspond to the mean value of each parents: A AN99,78,51; B Clery; C Alba; D AN94,414,52; E AN00,239,55; F AN03,338,56.

The F2 families, originated from back crossing of F1 - FVG, showed improvement in TPH values compared to parental values for all the three cross combinations. The mean values for all F2 families fell between their respective parents. Improvements were noticed for AN07,005 (2.40 \pm 0.08$)$ and AN07,003 (2.21 \pm 0.34$)$ while for AN07,004 $(1.95 \pm 0.04)$ no values were found above AN94,414,52, the parent with the highest TPH value.

Offspring from families AN07,009 (1.80 \pm 0.04$)$ and AN07,007 (1.74 \pm 0.04$)$, both with the selection AN99,78,51 as common parent, had lower TPH mean values than both parents. The unhelpful effect of AN99,78,51 as parent is clearly shown by the reduced mean and variation observed in the cross combination with AN00,239,55, the parent used because of the highest TPH content of its fruit.

\subsection{Offspring total distribution and family variation of fruit ACY content}

Fruit ACY contents of all offspring showed a non significant distribution (Fig. 5). The highest frequency corresponded with the mean values of 2 parents (Clery and AN00,239,55), while all the other parents had lower values. The peculiarity of this distribution is the high number of accessions (35 over 81) having fruit with an anthocyanin content that goes beyond the average population value.

The ANOVA analyses for ACY showed significant differences among cross combinations but with a different trend as observed for the distribution of TAC and TPH values. For this trait all families showed mean values going beyond the parental lines (Fig. 6).

Also for ACY the greatest improvement was found in fruit from the F3 inter specific cross combinations; in fact, the family mean values were higher than both the parents and the mean of all cross combinations. Fruit with the highest mean value of ACY was found for AN07,216 (6.47 \pm 0.19$)$, while the other families performed with lower mean values: AN07,215 (5.35 \pm 0.19$)$, AN07,006 (5.18 \pm 0.13$)$. For this trait, differently from the results encountered for TAC and TPH, the parent AN99,78,51 performed better in not affecting the performances of fruit ACY.

The ACY content among the F2 families showed improvements for all three cross combinations, even if their values were significantly lower than in the F3 families. Mean values of all families were higher than their respective parents, AN07,005 (4.87 \pm 0.19$)$ AN07,004 (4.79 \pm 0.14$)$ and AN07,003 (4.19 \pm 0.11$)$.

Positive results were also achieved in fruit analyzed from offspring originated by intra-specific crossing; in fact, also fruit of family AN07,009 (4.90 \pm 0.14$)$ showed average ACY values higher than the parental lines, while family AN07,007 (3.40 \pm 0.15$)$ behaved with a variation corresponding with the parental lines. 


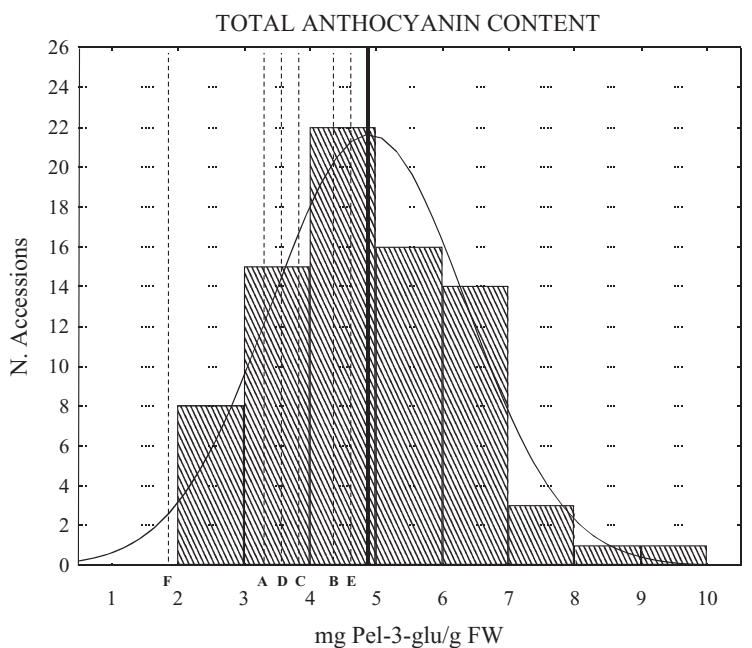

Fig. 5. Distribution detected for the fruit Anthocyanin Content of offspring originated from the eight cross combination and the Normal Distribution calculated with Kolmogorov-Smirnov test $d=0.042, p=$ n.s. $(p \leq 0.05)$. Letters correspond to the mean value of each parents: A AN99,78,51; B Clery; C Alba; D AN94,414,52; E AN00,239,55; F AN03,338,56.

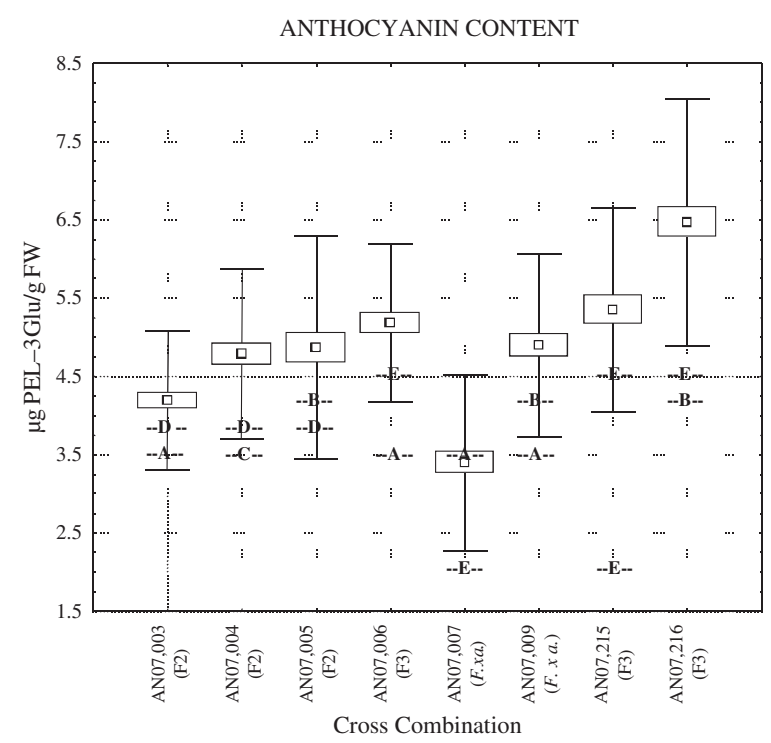

Fig. 6. Box and whisker Cross Combination Anthocyanin Content. Results are shown as mean value, Standard Error, Standard Deviation. Letters correspond to the mean value of each parents: A AN99,78,51; B Clery; C Alba; D AN94,414,52; E AN00,239,55; F AN03,338,56.

\subsection{Offspring and parents correlations among TPH, ACY and TAC}

Pearson correlation confirmed the high correlation between fruit TAC and TPH (Table 3), both for parents $(0.795)$ and offspring (0.739). No correlation was detected between TAC and ACY of fruit from parents' lines (0.072), while the same parameters showed a higher correlation value from fruit of the offspring (0.430). Parents and offspring showed low but similar correlation values between fruit TPH and ACY (Table 3). 
Table 3

Pearson correlation values calculated for the 3 nutritional parameters (total antioxidant capacity, total phenol and anthocyanin content) calculated for fruit sampled from the parents and from the offspring

\begin{tabular}{lccc}
\hline Antioxidant attributes & Total antioxidant capacity & Total phenol content & Anthocyanin content \\
\hline Pearson correlation among parents & & & $0.072^{\mathrm{ns}}$ \\
$\quad$ Total antioxidant capacity & - & - & $0.430^{* *}$ \\
Total phenol Content & & & - \\
Anthocyanin content & - & $0.739^{* *}$ & $0.419^{* *}$ \\
Pearson correlation among offspring & & - & $0.408^{* *}$ \\
Total antioxidant Capacity & & - \\
Total phenol content & & & - \\
Anthocyanin content & & &
\end{tabular}

ns, $*, * *$ Non significant, significant at $p \leq 0.05$ and $p \leq 0.01$ respectively.

\subsection{Parents and offspring variations and breeding progress}

The scatter-plot of parents and offspring fruit TAC and TPH has confirmed a high linear correlation among these values $\left(R^{2}=0.67\right)$ (Fig. 7) and allowed the identification of 10 new genotypes (black dots) combining the highest values of both TAC and TPH. Nine out of 10 of these genotypes belongs to the F2 back crossing (AN07,216 and AN07,215) and 1 to the F1 back crossing (AN07,005,51).

Comparing the distribution of the values detected from parents and offspring, TAC values of 51 accessions (64.6\%) were found to be higher than the mean value of the parent's $(21.63 \mu \mathrm{mol} \mathrm{TE} / \mathrm{g} \mathrm{FW})$ while 37 offspring (46.4\%) showed higher values compared to the total mean population $(23.28 \mu \mathrm{mol} \mathrm{TE} / \mathrm{g} \mathrm{FW})$. The overall offspring mean increased by $1.65 \mathrm{TE} / \mathrm{g} \mathrm{FW}$ compared to the parents' mean values (Fig. 7).

For TPH, 21 offspring (26.3\%) showed higher values compared to the parents' mean (2.55 mg GA/g FW) while 30 offspring (37.5\%) had higher values in comparison to the overall mean population $(2.28 \mathrm{mg} \mathrm{GA} / \mathrm{g} \mathrm{FW})$. As for TAC, the same 10 genotypes were selected also for the higher TPH of their fruit (Fig. 7).

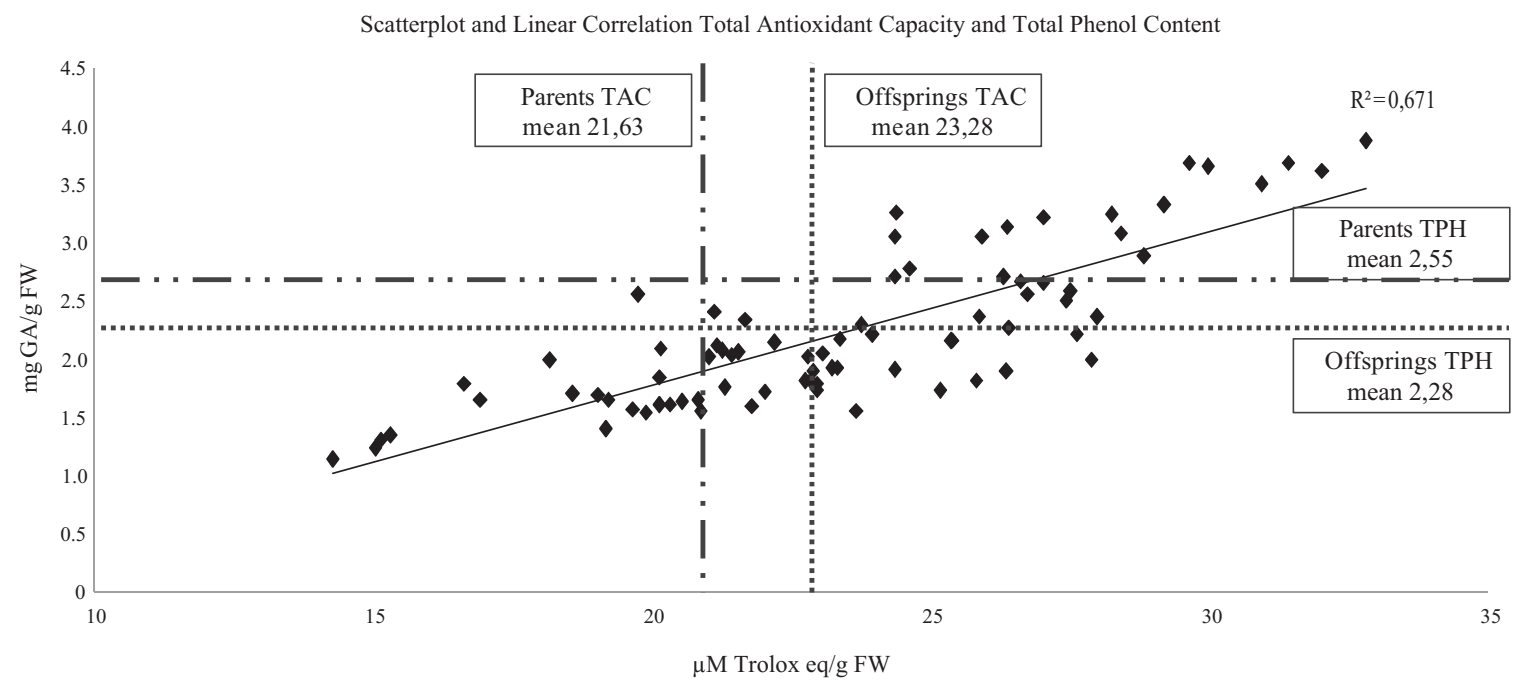

Fig. 7. Scatterplot distribution and linear correlation of Total Antioxidant Capacity( $\mu$ M Trolox eq/g FW) and Total Phenol Content (mg Gallic Acid/g FW) values detected from fruit of parents and offspring, compared with the corresponding means of all parents and all offspring for both characters (TAC, TPH). 


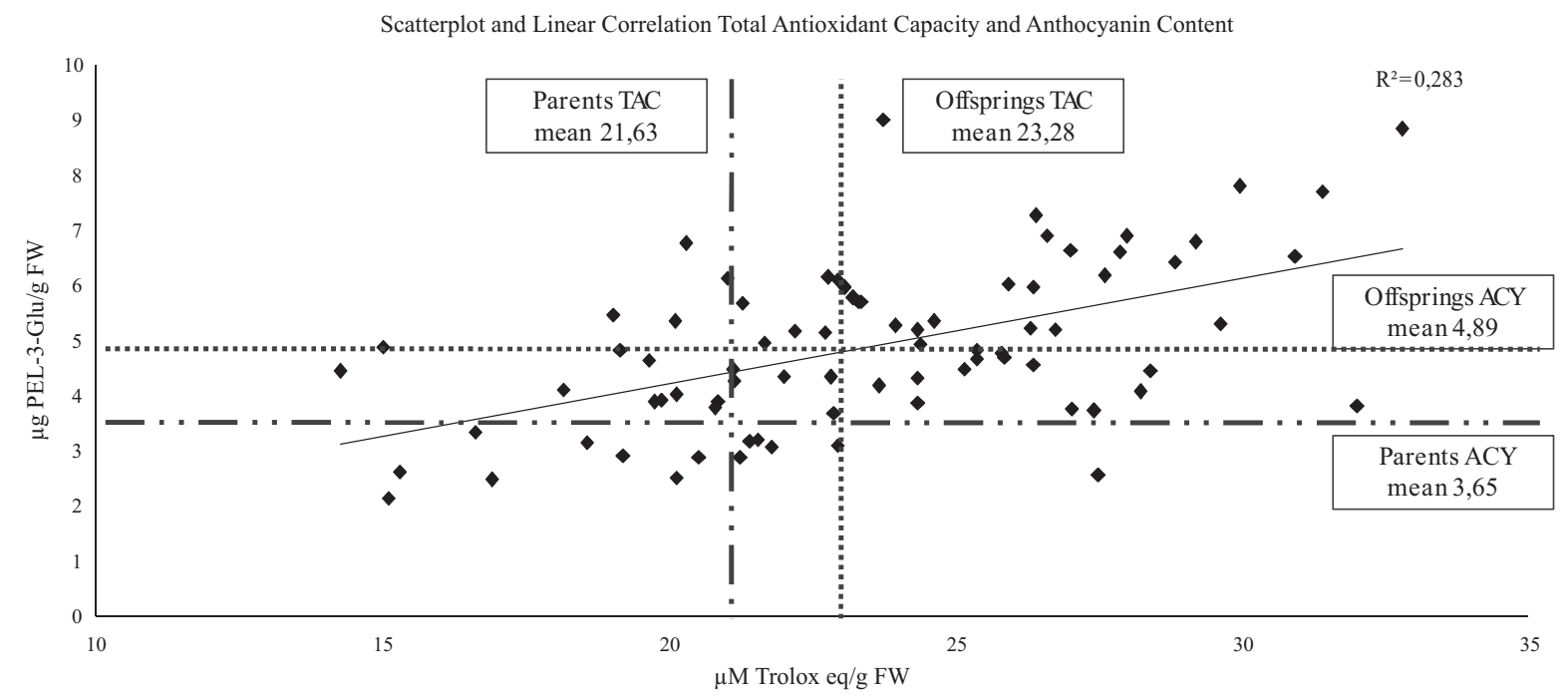

Fig. 8. Scatterplot distribution and linear correlation of Total Antioxidant Capacity( $\mu$ M Trolox Eq/g FW) and Anthocyanin Content ( $\mu \mathrm{g}$ PEL-3$\mathrm{Glu} / \mathrm{g} \mathrm{FW}$ ) values detected from fruit of parents and offspring, compared with the corresponding means of all parents and all offspring for both characters (TAC, ACY).

The scatterplot distribution of parents and offspring fruit TAC and ACY showed a low linear correlation among genotype $\left(R^{2}=0.28\right)$. ACY of 66 offspring $(82.5 \%)$ showed higher values compared to the parents' mean value (3.65 $\mu \mathrm{g}$ PEL-3-GLU/g FW), while 37 offspring (46.4\%) had higher values in respect to the overall population mean value (4.89 $\mu \mathrm{g}$ PEL-3-GLU/g FW).

\subsection{Breeding progress related to the type of cross combination}

The ANOVA for the effect of type of cross combination showed significant differences among the three types of crossing ( $p \leq 0.01$ ), for all nutritional traits taken into account for this study (Table 4 ).

Fruit of the F2 back-crossing combination had significantly higher mean values of TAC, TPH and ACY compared to fruits of F1 back-crossing combination and fruit originating from intra-specific crossing. Fruits from the F1 backcrossing had significantly higher values for the nutritional traits than fruit derived from intra-specific F. $x$ ananassa cross combinations (Table 4).

Table 4

ANOVA analysis on the effect of type of intra and inter-specific cross combinations on fruit nutritional quality parameters

\begin{tabular}{|c|c|c|c|c|c|c|}
\hline Crossing & \multicolumn{2}{|c|}{ TAC } & \multicolumn{2}{|c|}{$\mathrm{TPH}$} & \multicolumn{2}{|c|}{$\mathrm{ACY}$} \\
\hline F3 Backcross & \multicolumn{2}{|c|}{$24.70 \pm 0.44 c$} & \multicolumn{2}{|c|}{$2.59 \pm 0.06 \mathrm{c}$} & \multicolumn{2}{|c|}{$5.72 \pm 0.11 \mathrm{c}$} \\
\hline F2 Backcross & \multicolumn{2}{|c|}{$23.65 \pm 0.23 b$} & \multicolumn{2}{|c|}{$2.18 \pm 0.03 b$} & \multicolumn{2}{|c|}{$4.59 \pm 0.09 b$} \\
\hline$F \times a$. Crossing & \multicolumn{2}{|c|}{$20.78 \pm 0.29 a$} & \multicolumn{2}{|c|}{$1.77 \pm 0.03 \mathrm{a}$} & \multicolumn{2}{|c|}{$4.18 \pm 0.12 \mathrm{a}$} \\
\hline ANOVA & $F$ & $p$ Level & $F$ & $p$ Level & $F$ & $p$ Level \\
\hline Crossing & 31.221 & $0.000^{* *}$ & 72.804 & $0.000 * *$ & 55.825 & $0.000 * *$ \\
\hline
\end{tabular}

Means values followed by different letters are significantly different. Test SNK $p \leq 0.05$.

$\mathrm{NS}, *, * *$ Non significant, significant at $p \leq 0.05$ and $p \leq 0.01$ respectively. 


\section{Discussion and conclusions}

The evaluation carried out on the genetic pool which originated from a breeding program including $F$. $x$ ananassa intra-specific crossing and $F . x$ ananass $a$ and $F V G$ inter-specific backcrossing confirmed the significant highest increase of the main nutritional traits (TAC, TPH and ACY) of fruit in families originated from FVG inter-specific back-crossing. In fact, fruit of F3 family AN07,215 and AN07,216 showed the highest values for all nutritional parameters evaluated. Fruit of F2 families' AN07,004 and AN07,005 also emerged for their nutritional values which were even higher than fruit sampled from plants originated from intra-specific cross combinations. Almost all these new genotypes also showed an improvement of other important agronomic traits such as fruit size and firmness and plant productivity, very close to commercial requirements. The observed genetic improvement of the new selected genotypes, needs confirmation with further evaluation in order to better determine the combinations of the increased fruit nutritional values with the other important agronomic traits requested commercially. Furthermore, the real increase in human health values also needs validation, like the one already carried out for other strawberry varieties [22].

The results on this new breeding material confirm the usefulness of FVG as a wild parent to increase strawberry fruit nutritional quality $[4,5]$, but this result is strictly related to the use of proper combining parents. In fact, offspring originated from F2 (AN07,003) and F3 (AN07,006) back crossed using AN99,078,51 as common parent (father) produced the lowest fruit TAC mean values, as well as TPH and ACY, in comparison with all the cross combinations. The lowest fruit nutritional quality was also detected from AN07,007 the intra specific cross combination also including AN99,078,51 as parent (father). This advanced selection was used because of the interesting traits of the fruit such as firmness, high soluble solids, red color and also aroma. Unfortunately, from these results the important fruit traits of this selection do not seem to be properly associated with a genetic base which is useful for the achievement of new genotypes with a proper combination of high values of all quality and nutritional parameters. Among the 2 F. $x$ ananassa cross combinations, both with AN99,078,51 as common father, the family AN07,009 differed with better improvement of the fruit nutritional quality, probably due to the positive effect of Clery as second parent. The positive effect of Clery in increasing fruit nutritional quality was also confirmed by the highest mean values observed when used as parent in F2 (AN07,005) and F3 (AN07,216) backcrossing.

The results obtained in this work show that the use of the wild genotype of $F$. virginiana spp. glauca in inter-specific crossing and its consequential back crossing is useful in improving the nutritional quality of fruit. Improvements of these traits can be achieved also by programming $F$. $x$ ananassa intra-specific crosses. The two types of combination programs (inter-specific backcrosses and intra-specific crosses) can be used for the improvement of strawberry nutritional quality, but the success of the program is strictly related to the combining attitude of the different parents. For this reason, this type of experiment can be considered important in order: (i) to produce new genotypes and (ii) to identify cultivars and selections able to perform as most useful parents to be used in a strawberry breeding program aimed at producing new improved varieties for a better combination of the agronomic and overall fruit quality traits.

\section{References}

[1] R. Azodanlou, C. Darbellay, J. Luisier, J. Villettaz and R. Amadò, Quality assessment of strawberry (Fragaria Species), Journal of Agricultural and Food Chemistry 51 (2003), 715-721.

[2] M. Battino, J. Beekwilder, B. Denoyes-Rothan, M. Laimer, G.J. McDougall and B. Mezzetti, Bioactivities of berries relevant to human health, Nutrition Reviews 67 (2009), S145-S150.

[3] R.S. Bringhurst and V. Voth, Origin and evolutionary potentiality of the day-neutral trait in octoploid Fragaria, Genetics 90 (1978), 510.

[4] F. Capocasa, J. Diamanti, S. Tulipani, M. Battino and B. Mezzetti, Breeding strawberry (Fragaria $x$ ananassa Duch) to increase fruit nutritional quality, Biofactors 34 (2008), 67-72.

[5] F. Capocasa, J. Scalzo, B. Mezzetti and M. Battino, Combining quality and antioxidant attributes in the strawberry: The role of the genotype, Food Chemistry 111 (2009), 872-878.

[6] A.M. Connor, J.J. Luby, C.B.S. Tong, C.E. Finn and J.F. Hancock, Variation and heritability estimates for antioxidant activity. Total phenolic content, and anthocyanin content in blueberry progenies, Journal of American Society Horticultural Science 1 (2002), 82-88.

[7] A.M. Connor, M.J. Stephens, H.K. Hall and P.A. Alspach, Variation and heritabilities of antioxidant activity and total phenolic content estimated from a red raspberry factorial experiment, Journal of American Society Horticultural Science 3 (2005), 130. 
[8] N. Deighton, R. Brennan, C. Finn and H.V. Davies, Antioxidant properties of domesticated and wild Rubus species, Journal of the Science of Food and Agriculture $\mathbf{8 0}$ (2000), 1307-1313.

[9] D. Della Penna, Plant metabolic engineering, Plant Physiology 125 (2001), 160-163.

[10] M. Giusti and R.E. Wrolstad, Characterization and measurement of anthocyanins by UV-visible spectroscopy, Current Protocols in Food Analytical Chemistry (2001), F1.2.1-F1.2.13.

[11] J.F. Hancock, J.J. Luby, A. Dale, P.W. Callow, S. Serce and A. El-Shiek, Utilizing wild Fragaria virginiana in strawberry cultivar development: Inheritance of photoperiod sensitivity, fruit size, gender, female fertility and disease resistance, Euphytica 126 (2002),177-184.

[12] K.J. Meyers, C.B. Watkins, M.P. Pritts and R.H. Liu, Antioxidant and antiproliferative activities of strawberries, Journal of Agricultural and Food Chemistry 51 (2003), 6887-6892.

[13] N.J. Miller, C. Rice-Evans and M.J. Davis, A novel method for measuring antioxidant capacity and its application to monitoring the antioxidant status in premature neonates, Clinical Science 84 (1993), 407-412.

[14] M.E. Olsson, J. Ekvall, K. Gustavsson, J. Nilsson, D. Pillai, I. Sjoholm, U. Svensson, B. Akesso and M.G. Nyman, Antioxidants, low molecular weight carbohydrates, and total antioxidant capacity in strawberry (Fragaria x ananassa), Journal of Agricultural Food Chemistry 52 (2004), 2490-2498.

[15] R.L. Prior, G. Cao, A. Martin, N. Sofic, J. McEwen, C. O’Brien, N. Lischner, M. Ehlenfeldt, W. Kalt, G. Krewer and C.M. Mainland, Antioxidant capacity as influenced by total phenolic and anthocyanin content, maturity and variety of Vaccinium Species, Journal of Agricultural Food Chemistry 46 (1998), 2686-2693.

[16] A.R. Proteggente, A.S. Pannala, G. Pagana, L. Van Buren, E. Wagner, S. Wiswman, F. Van De Put, C. Dacombe and C.A. Rice-Evans, The antioxidant activity of regular consumed fruit and vegetables reflects their phenolic and vitamin C composition, Free Radical Research 36 (2002), 217-233.

[17] R. Re, N. Pellegrini, A. Proteggente, A. Pannala, M. Yang and C. Rice-Evans, Antioxidant activity applying an improved ABTS radical cation decolorization assay, Free Radical Biology \& Medicine 26 (1999), 1231-1237.

[18] J. Scalzo, A. Politi, B. Mezzetti and M. Battino, Plant genotype affects total antioxidant capacity and phenolic contents in fruit, Nutrition 21 (2005), 207-213.

[19] Y. Shiow, L. Wang and S. Kim, Antioxidant capacity and flavonoid content in wild strawberries, Journalof American Society of Horticutural Science 132 (2007), 629-637.

[20] K. Slinkard and V.L. Singleton, Total phenol analysis: Automation and comparision with manual methods, American Journal of Enology and Viticulture 28 (1977) 49-55.

[21] S. Tulipani, B. Mezzetti, F. Capocasa, S. Bompadre, J. Beekwilder, C.H.R. De Vos, E. Capanoglu, A. Bovy and M. Battino, Antioxidants, phenolic compounds, and nutritional quality of different strawberry genotypes, Journal of Agricultural and Food Chemistry 56 (2008), 696-704.

[22] S. Tulipani, B. Mezzetti and M. Battino, Impact of strawberries on human health: Insight into marginally discussed bioactive compounds for the Mediterranean diet, Public Health Nutrition 12 (2009), 1656-1662.

[23] S. Tulipani, S. Romandini, F. Busco, S. Bompadre, B. Mezzetti and M. Battino, Ascorbate, not urate, modulates the plasma antioxidant capacity after strawberry intake, Food Chemistry 117 (2009), 181-188.

[24] S.Y. Wang and K.S. Lewers, Antioxidant capacity and flavonoid content in wild strawberries, Journal of American Society of Horticultural Science 132 (2007), 629-637.

[25] S.Y. Wang, W. Zheng and G.J. Galletta, Cultural system affects fruit quality and antioxidant capacity in strawberries, Journal of Agricultural Food Chemistry 50 (2002), 6534-6542.

[26] S.Y. Wang and H.S. Lin, Antioxidant activity in fruits and leaves of blackberry, raspberry, and strawberry varies with cultivar and developmental stage, Journal of Agricultural Food Chemistry 48 (2000), 140-146.

[27] W. Zhen and S.Y. Wang, Oxygen radical absorbing capacity of phenolics in blueberries, cranberries, chokeberries, and lingonberries, Journal of Agricultural and Food Chemistry 51 (2003), 502-509. 